Am. J. Nephrol. 1982;2:321

\title{
Medical Art
}

\begin{tabular}{|l|l|l}
\hline D. & Casellas \\
\hline
\end{tabular}

S. Scanning electron micrograph of a glomerular capillary loop from a rat injected with labeled microspheres $(7-11 \mu \mathrm{m})$ into the left ventricle for renal blood flow determination. Part of the capillary wall is cracked revealing an impacted microsphere with platelets adherent to its surface. $\times 3,000$. 